\title{
Study of Cross-Platform Technologies for Data Delivery in Regional Web Surveys in the Education
}

\author{
Evgeny Nikulchev ${ }^{1}$, Dmitry Ilin ${ }^{2}$ \\ Vladimir Belov ${ }^{3}$, Pavel Pushkin ${ }^{4}$ \\ MIREA-Russian Technological \\ University, Russia
}

\author{
Pavel Kolyasnikov ${ }^{5}$ \\ Russian Academy of Education \\ Russia
}

\author{
Sergey Malykh ${ }^{6}$ \\ Psychological Institute of Russian \\ Academy of Education \\ Russia
}

\begin{abstract}
Web-surveys are a popular form of collecting primary data from various studies. However, mass regional polls have their own characteristics, including the following: it is necessary to take into account various platforms and browsers, as well as the speed of networks, if rural areas remote from large centers are involved in the polls. Ensuring guaranteed data delivery in these conditions should be the right choice of technology for the implementation of surveys. The paper presents the analysis results of the technologies sustainability to various regional conditions for web survey conducted within one week at schools throughout the Russian Federation. The survey involved 20000 real educators. The paper describes the technologies used and provides information about browsers and operation systems used by respondents. The absence of failures in data delivery confirms the effectiveness of the solutions.
\end{abstract}

Keywords-Web-surveys; mass regional polls; various platforms and browsers; cross-platform technologies

\section{INTRODUCTION}

An adequate choice of information technology ensures a guaranteed quality of primary data delivery and user convenience in mass regional surveys.

The research was conducted using the digital platform [1] for large-scale psychological research DegitilPsyTool.ru [2], developed by the Russian Academy of Education. The platform is designed to collect large amounts of data using web technologies [3], meets the requirements of the legislation of the Russian Federation for the functioning of information systems.

The digital platform is a tool for collecting and storing research data, which are the basis for the formation of methods, analytical materials, guidelines, at the territorial, regional and federal levels.

Primary data collection is carried out using web interfaces [4] of the described platform. Database is being formed based on surveys at schools [5]. These data are going to be available for analysis by multidisciplinary research teams. The methods used to conduct research are integrated into the digital platform after careful study and become available for expert analysis. Long-term data storage is provided in conjunction with the methods used in the Data Center of Russian Academy of Education. This will allow to track the dynamics of changes in indicators, their characteristics, to make selective analysis according to regional characteristics, to consider factors influencing factors on the value of indicators, as well as their change over time.

Digital Psychological Tools platform is developed as an ever-evolving information environment with a set of tools for conducting research at all levels, consolidating diverse research groups and ensuring interaction between them.

Data collection tools are aimed at followings: data collecting in educational institutions and samples of any size; collecting population research data; collecting longitudinal research data; collecting research data on experimental techniques. Tools were created for formatting new techniques and use the capabilities of the existing base of proven, reliable methods of psychological research.

The platform tools allow conducting a simultaneous survey of a large number of schools, including through the use of technology for simultaneous work both online and offline, followed by the transfer of results.

The platform is developed to comply with the requirements of the legislation of the Russian Federation for data storing and processing.

Big data storage tools meet today's technological challenges. Based on new information technologies and data structuring for processing large amounts of data, the followings have been formed: a multi-level data storage system that provides quick access to frequently used data, a poorly structured storage form that allows you to store data along with the methods used; a virtualization system for computing resources, which allows for simultaneous development and operation of the platform; a backup system that ensures the integrity and safety of data, as well as a system of customizable switching network interaction with databases, to ensure the operational interaction of applications and analyticalinformation systems with databases.

The paper consists of 2 parts. The first part presents the results of the analysis of the sustainability of technologies to various regional conditions in conducting a web survey completed within one week throughout the Russian Federation at schools. The survey involved 20,000 real educators. The first part discusses the issues of creating a cross-platform interface and presents the results on the use of browsers in conducting mass research in the education system on the territory of the Russian Federation. The second part describes the solution for collecting data in a browser and then packing it into an archive, 
which ensures independence from the communication channel during the survey completing.

\section{CROSS-PlatFORM RESEARCH}

For web-based software implementation of the test survey, an internal standard for the presentation of the test in a structured form has been developed. It allows using a special algorithmic structure to create a psychological test interface based on elements that have been developed and tested on various devices and OS. The presence of the standard makes it possible to develop an interactive designer of quiz tests to automate the creation of new tests.

Based on the analysis of functional requirements and limitations related to the survey, it was found that the most effective form of intra-platform presentation is the use of JSON (JavaScript Object Notation) format [6,7]. To control the structure of JSON documents [8, 9], JSON Schema tools are used.

In the platform, each research methodology consists of automatically generated surveys (for school principals and psychologists). The software implementation of each survey is a web interface for interviewing respondents. At the same time, an important feature is the need for cross-platform functioning of interfaces [10], which makes it possible to conduct polls on a wide range of devices, popular browsers and operation systems like Windows, Linux, MacOS, iOS, Android. The used intra-platform standard for describing survey elements will allow the automatic generation of an interface [11] in various software environments.

Generated on the basis of the survey description standard [12], the web interface has been tested on various devices, operation systems and browsers. The approach used is crossbrowser [13] and allows the interface to work and to display in all popular browsers with a certain adaptation in real time. A schematic example of the display of the user interface for various types of devices is shown in Fig. 1.

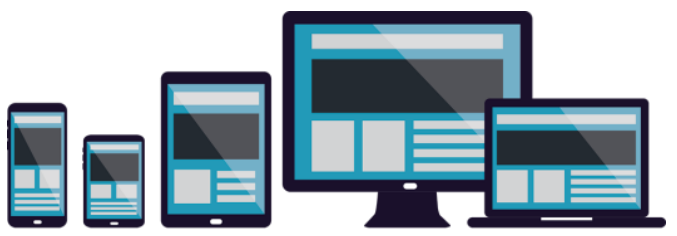

Fig. 1. An Example of Adapting an Interface for Various Types of Devices.

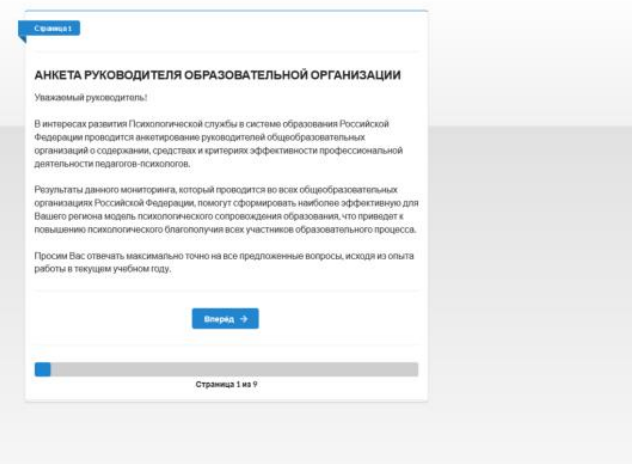

Fig. 2. The Initial Page of the School Head's Surveys.
Fig. 2 to 4 show screenshots of the school heads surveys.

Fig. 5 to 7 shows screenshots of the survey pages.

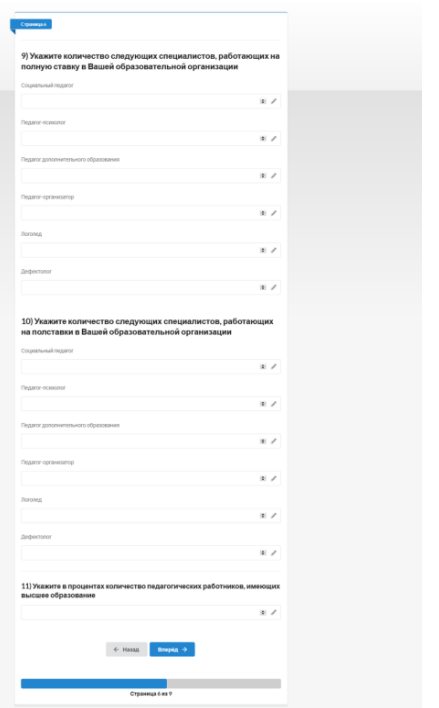

Fig. 3. An Example of the School Head's Survey Interface.

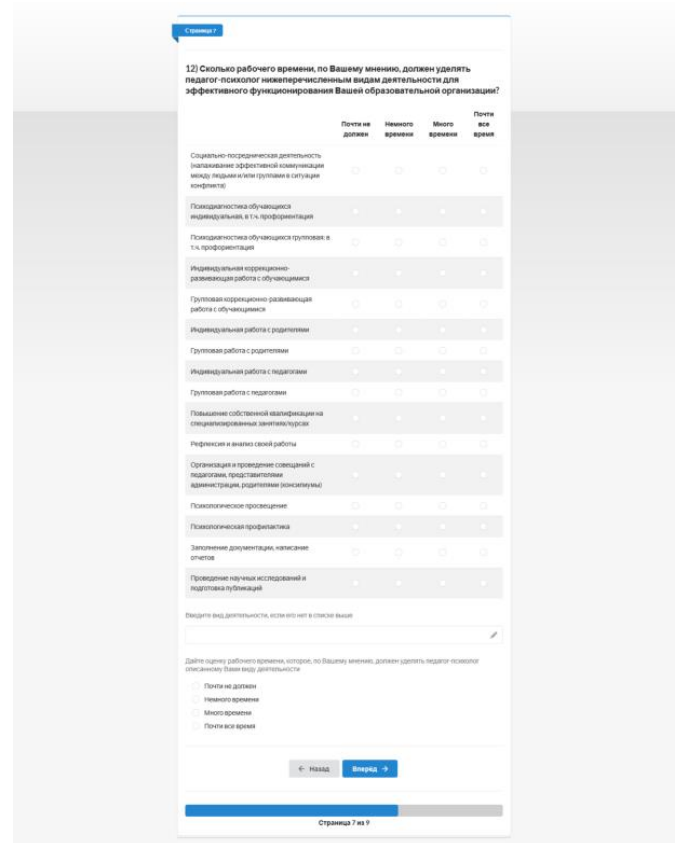

Fig. 4. An Example of the Interface for School Heads.

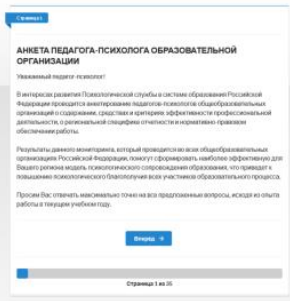

Fig. 5. The Initial Page of the Psychologist Profile. 


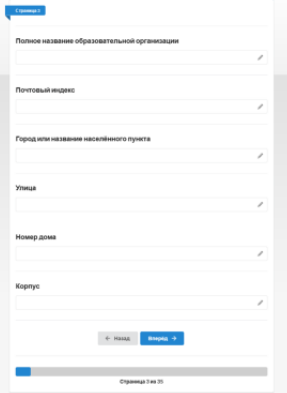

Fig. 6. An Example of the Profile Interface for the Psychologist.

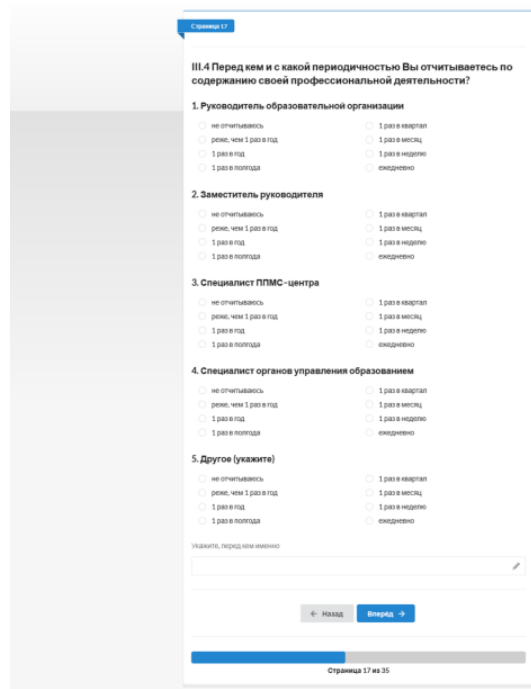

Fig. 7. An Example of the Profile Interface for the Psychologist.

As a monitoring result, information was obtained on the operation systems and browsers types used for survey. The results obtained indicate the effectiveness of the selected technologies, since all surveys were successfully completed without distortion of the images from the respondents. Fig. 8 shows the main operation systems, in Fig. 9 shows browsers used to fill out surveys at schools during a mass survey across Russian Federation.

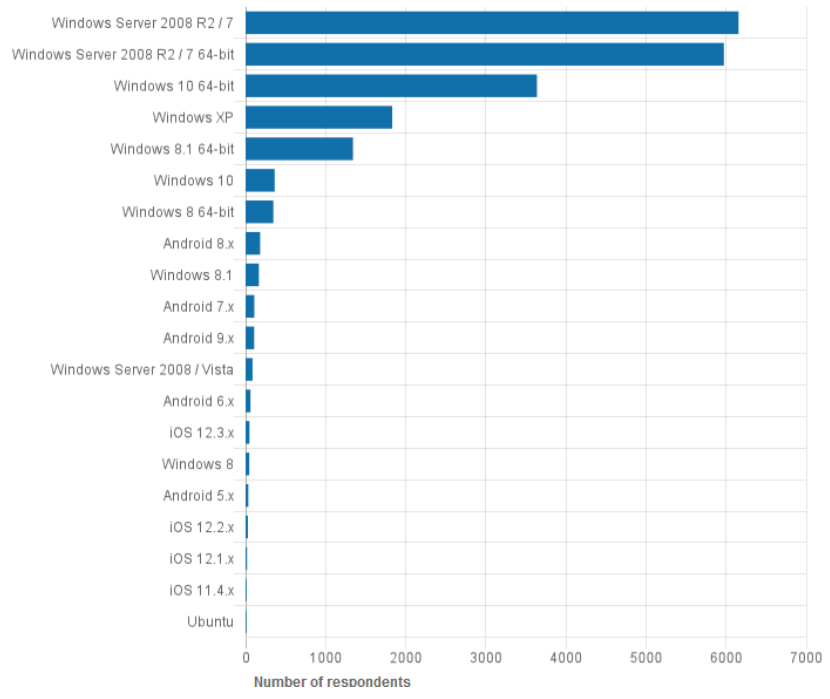

Fig. 8. Operation Systems used to Fill out Survey.

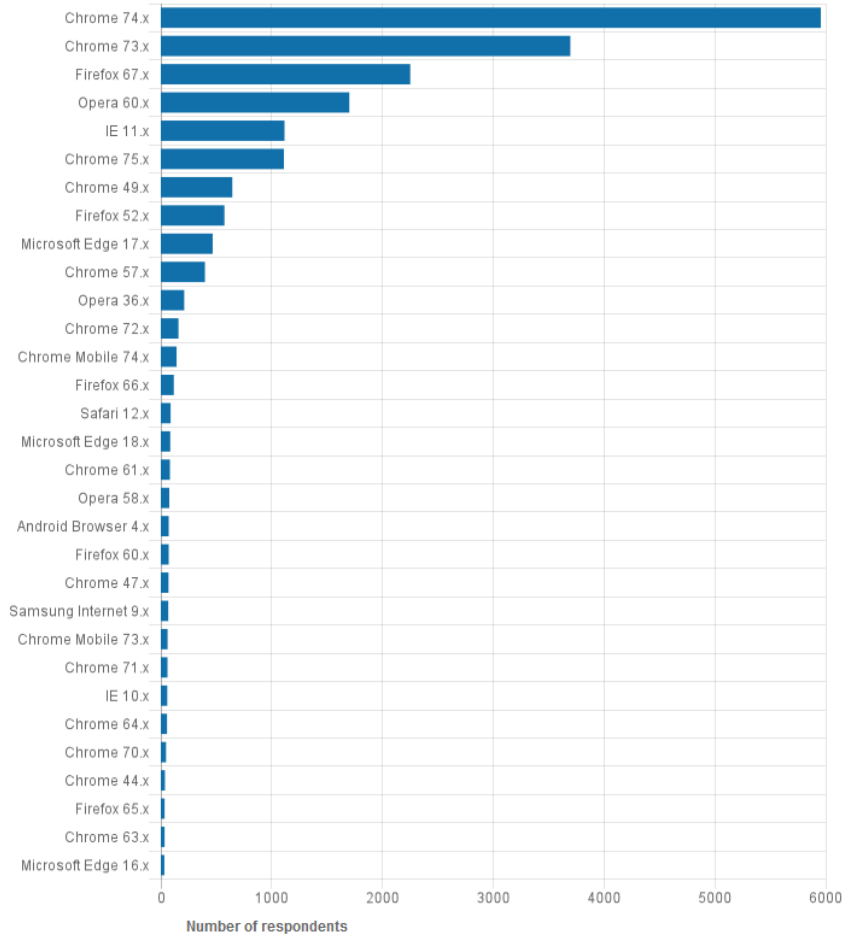

Fig. 9. Browsers used to Fill out Survey.

\section{THE USE OF THE ARCHIVING FOR ENSURING GUARANTEED DATA DELIVERY}

One of the monitoring tasks is to ensure functioning, independent of the quality of the communication channel. This problem is solved due to the following logic. Firstly, the archive, which contains necessary files (such as images) and the survey structure based on the JSON schema, is being loaded onto the client side. Then, the sever receives not archived JSON file with the survey results (it contains the numbers of questions and entered data).

It is assumed that the archive is a complete package that is transmitted to the client browser along with the survey format and other data necessary for data collection. All system data must be downloaded before the test starts and its integrity check is carried out (checksum). To ensure control, it is more convenient to transfer data to the browser in the form of an archive, since it will be necessary to download only one file and check only its checksum. If you use the standard approach and upload multiple files separately, you will have to verify the checksums for each of them separately, and on the server side you will need to organize additional storage.

Using client-side archives changes the standard approach for working with files. In the standard approach, the address of the actual location of the requested resource is indicated in HTML, for example, an image that will be downloaded by HTTP request and displayed automatically by the browser. In the case of using archives, all actions will need to be done programmatically: download it in its entirety, unpack it and only then use it in HTML. In addition, the browser may require more computing resources and computer RAM to work with the archive, since it will not be able to directly optimize the loading, saving and caching downloaded images and files. 
As an archive, the preferred option is using the zip format, which is supported by a large number of operation systems and programs. This will allow to pack and unpack the contents of the survey without the need for additional software. In this regard, it will be most appropriate to use the JSZip library [14], which has an API for working with zip archives.

JSZip is a JavaScript library for reading, creating and editing zip files and is distributed on the basis of two licenses MIT or GPLv3. According to the documentation [15], JSZip is guaranteed to support files up to $10 \mathrm{MB}$ in size, does not support data encryption and multi volumes, uses only UTF-8. The library works in all modern browsers, such as Firefox, Google Chrome, Opera, Internet Explorer 10+ and Edge. In Internet Explorer 9 and below, JSZip does not work well and may crash when working with large files, as these browser versions do not support typed arrays and the compression algorithm will use regular arrays.

A number of tests were performed to evaluate the capabilities of the JSZip library.

Testing Cyrillic characters. Archives that were created on Mac OS are read normally, while archives that were created on Windows have incorrectly encoded file names. Due to the fact that on Mac OS, the encoding is UTF-8, and on Windows, most likely, cp1251. The compression level does not affect the ability to read archives; only the unpacking time and CPU load may change, but only slightly. Encrypted archives are not supported by the library and cannot be opened. Supported compression method Deflate, other methods work will not be read, due to the use of the pako library.

Testing bulk archives. Various computers and smartphones were taken to test the JSZip library. According to the measurements, it is clear that the time of unpacking a large archive is not critical even on a mobile device.

The finished survey for the psychological platform is a complete ZIP archive. For preparing a test in the specified format, ZIP archiving should be used without compression and without setting a password.

The archive should contain the index.json file. This file should be executed strictly in accordance with the specification below. For a typical case, the following archive structure is proposed:

$$
\begin{aligned}
& \text { index.json } \\
& \text { resources/ } \\
& \text { question_1/ } \\
& \text { image_1.jpg } \\
& \text { image_2.jpg } \\
& \text {... } \\
& \text { question_2/ } \\
& \text { image_1.jpg } \\
& \text { image_2.jpg }
\end{aligned}
$$

In addition to the mandatory presence of the index.json file, there are the following limitations of the psychological test downloaded to the archive platform:

- archive size: no more than 10 megabytes;

- archive format: ZIP;

- compression: absent;

- password: none;

- file names: case sensitive.

Due to the ever-increasing complexity of tools and technologies in web development, the task arises of preparing the client part of the project for its proper operation and display in the user's browser. For its implementation, assembly systems are used that automate the process of preparing code and resources of the client part of the web application. The main objective of automation systems for assembling client web applications is to combine the same type of modules and automate routine tasks.

The main tasks implemented by the assembly systems can be distinguished:

- web language compilation (CoffeeScript, TypeScript, Babel) [16, 17];

- compilation of CSS preprocessor (LESS, SASS, Stylus) and postprocessors (PostCSS, Rework) [18, 19];

- modular system implementation JavaScript (AMD, CommonJS) [20];

- code minification and obfuscation;

- preparing web resources (image compression, deleting meta data, creating sprites, preparing fonts, etc.);

- checking the code for quality, i.e. code linting (ESLint, JSLint, JSCS, JSHint) [21-23].

As a solution for building the client part of the web application, Webpack was used [24], which is a modern solution that provides high speed and relevant tools for the developer. Webpack, unlike the Gulp and Grunt counterparts, offers much more features by default and covers most of the required build tasks. However, it is worth noting that Gulp and Grunt are task managers for Node.js, and not a complete build system like Webpack. To create a build process on Gulp and Grunt, it is needed to write manual build tasks.

To implement the functionality that is not available in the standard Webpack package, the following extensions were applied:

- ExtractTextPlugin. It is used to extract the text of the processed module and save to an external file. Required when processing style sheets.

- OptimizeCssAssetsPlugin. It implements the functionality to minimize the resulting CSS markup code.

- CopyWebpackPlugin. It implements the functionality of copying individual files or structural units. 
- HotModuleReplacement. It implements a quick module replacement mechanism. This mechanism significantly increases development speed and is used when tracking changes to apply them.

- ProvidePlugin. It imports dependencies common to the entire project.

- UglifyJsPlugin. It implements the functionality for minimizing JavaScript code.

- ConfigWebpackPlugin. It combines environment and internal configuration.

- Optimize.CommonChunk. It separates code sections, which are common for several modules, into a separate file.

In addition to extensions, the project also involved external loaders that process the input data, since the functionality, they implement, cannot be implemented using standard Webpack tools:

- file-loader. Arbitrary input file processing.

- babel-loader. Compilation of JavaScript ES6 code in compatible with most browsers ECMAScript 5;

- css-loader. CSS code processing;

- sass-loader. Convert SCSS and SASS code to CSS;

- ng-cache-loader. Provides support for HTML templates;

- image-webpack-loader. Image processing and compression.

The results of monitoring at schools in Russia demonstrated the effectiveness of the selected methods and information technologies. Fig. 10 shows the distribution of the rendering time of the survey on the client device, which confirms the advisability in using the developed technology for transmitting data in the form of an archive.

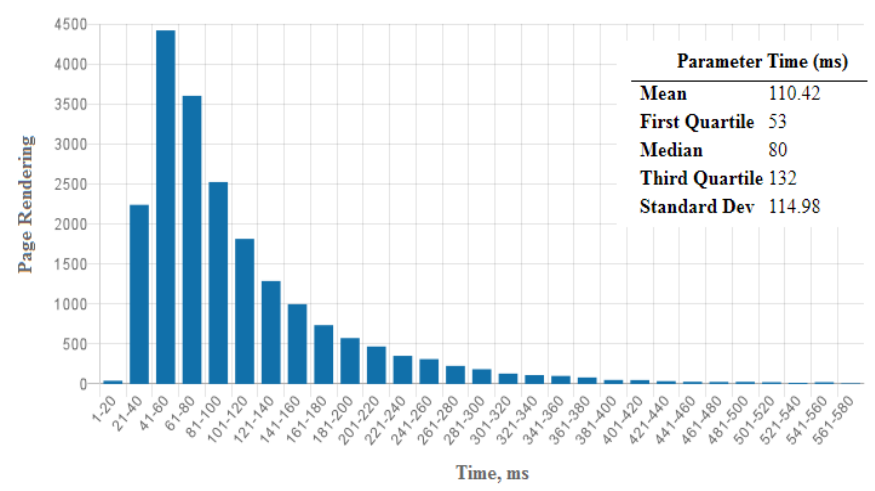

Fig. 10. Distribution Characterizing the Time of Rendering Survey Pages.

\section{CONCLUSION}

The survey involved 20,000 real school heads from municipal and village schools throughout the country. The absence of failures in data delivery using various operation systems and browsers confirms the effectiveness of the solutions.

Thus, the selected web-based survey tools based on the Digital Platform for Interdisciplinary Psychological Research, as a result of monitoring, have demonstrated their effectiveness and prospects for conducting federal-level research at schools.

\section{ACKNOWLEDGMENTS}

The study was performed as part of the state task of the Ministry of science and height education of Russian Federation, project 25.13253.2018 / 12.1 "Development of the technological concept of the Data Center for Interdisciplinary Research in Education".

\section{REFERENCES}

[1] R. D. Hays, H. Liu, \& A. Kapteyn, "Use of Internet panels to conduct surveys," Behavior research methods, vol. 47, no. 3, pp. 685-690, 2015.

[2] I. Zakharov, E. Nikulchev, D. Ilin, V. Ismatullina, \& A. Fenin, "Webbased platform for psychology research," ITM Web of Conferences, vol. 10, p. 04006, 2017.

[3] E. Nikulchev, D. Ilin, P. Kolyasnikov, I. Zakharov, \& S. Malykh, "Programming technologies for the development of web-based platform for digital psychological tools," International Journal of Advanced Computer Science and Applications, vol. 9, no. 8, pp. 34-45, 2018.

[4] F. Keusch, "Why do people participate in Web surveys? Applying survey participation theory to Internet survey data collection," Management review quarterly, vol. 65, no. 3, pp. 183-216, 2015.

[5] M. Das, P. Ester, \& L. Kaczmirek (ed.), "Social and behavioral research and the internet: Advances in applied methods and research strategies," Routledge, 2018.

[6] J. Xin, C. Afrasiabi, S. Lelong, ..., \& C. Wu, "Cross-linking BioThings APIs through JSON-LD to facilitate knowledge exploration," BMC bioinformatics, vol. 19, no. 1, p. 30, 2018.

[7] S. Brahmia, Z. Brahmia, F. Grandi, \& R. Bouaziz, "A Disciplined Approach to Temporal Evolution and Versioning Support in JSON Data Stores," In Emerging Technologies and Applications in Data Processing and Management, IGI Global, 2019, pp. 114-133.

[8] A. Agocs, \& J. M. Le Goff, "A web service based on RESTful API and JSON Schema/JSON Meta Schema to construct knowledge graphs," In 2018 International Conference on Computer, Information and Telecommunication Systems (CITS), IEEE, 2018, pp. 1-5.

[9] J. L. C. Izquierdo, \& J. Cabot, "JSONDiscoverer: Visualizing the schema lurking behind JSON documents," Knowledge-Based Systems, vol. 103, pp. 52-55, 2016.

[10] M. Jasim, "Building cross-platform desktop applications with electron," Packt Publishing Ltd, 2017.

[11] E. Nikulchev, D. Ilin, P. Kolyasnikov, and A. Kosenkov, "E-learning Tools on the Healthcare Professional Social Networks," International Journal of Advanced Computer Science and Applications, vol. 10, no. 4, p. 29-34, 2019.

[12] V.K. Grigoriev, A.S. Ilyushechkin, \&M.A. Ovchinnikov, "Quality assessment of a user interface based on the mental time of performing the user tasks of the subject area," Russian Technological Journal, vol. 7, no. 1, pp. 38-47, 2019. 
[13] E. Yigitbas, A. Anjorin, I. Jovanovikj, ..., \& G. Engels, "Usability Evaluation of Model-Driven Cross-Device Web User Interfaces," In International Conference on Human-Centred Software Engineering, 2018, pp. 231-247.

[14] J. V. de Figueiredo Leite, J. M. X. N. Teixeira, \& V. Teichrieb, "Optimizing 3D object visualization on the web," International Conference on Virtual, Augmented and Mixed Reality, 2016, pp. 287298.

[15] S. Knightley, D. Duponchel, F. Buchinger, \& A. Afonso, "JSZip," Oracle, $2016 \quad$ (https://docs.oracle.com/cd/E80480_01/English/ licensing_information/helpmain.htm?toc.htm?103589.htm).

[16] T. Burnham, "Coffeescript: accelerated Javascript development," Pragmatic Bookshelf, 2015.

[17] Y. Fain, \& A. Moiseev, "Angular 2 Development with TypeScript," Manning Publications Co., 2016.
[18] F. Marani, “Adding CSS/JavaScript to Our Django Project," In Practical Django 2 and Channels 2. Apress, Berkeley, CA, 2019, pp. 179-203.

[19] Libby A. Mastering PostCSS for Web Design. - Packt Publishing Ltd, 2016.

[20] U. Campos, G. Smethurst, J. P. Moraes, R. Bonifácio, \&, G. Pinto, "Mining rule violations in JavaScript code snippets," In Proceedings of the 16th International Conference on Mining Software Repositories, 2019, pp. 195-199.

[21] K. F. Tómasdóttir, M. Aniche, \& A. Van Deursen, "The adoption of javascript linters in practice: A case study on eslint," IEEE Transactions on Software Engineering, 2018.

[22] D. Crockford, "Jslint: The javascript code quality tool," 2011 (http://www.jslint.com).

[23] A. Mardan, "Getting Node. js Apps Production Ready," Practical Node.js. Apress, Berkeley, CA, 2018, pp. 331-364.

[24] T. Koppers, "Webpack: Module bundler," 2016. 